\title{
Management of screwdriver-induced penetrating brain injury: a case report
}

\author{
Jia Shi, Yumin Mao, Jiachao Cao and Bo Dong ${ }^{*}$
}

\begin{abstract}
Background: Penetrating brain injury (PBI) can be caused by several objects ranging from knives to chopsticks. However, an assault with long and electric screwdriver is a peculiar accident and is relatively rare. Because of its rarity, the treatments of such injury are complex and nonstandardized.

Case presentation: We presented a case of a 54-year-old female who was stabbed with a screwdriver in her head and accompanied by loss of consciousness for $1 \mathrm{~h}$. Computer tomography (CT) demonstrated that the screwdriver passed through the right zygomatic bone to posterior cranial fossa. Early foreign body removal and hematoma evacuation were performed and the patient had a good postoperative recovery.

Conclusions: In this study, we discussed the clinical presentation and successful management of such a unique injury caused by a screwdriver. Our goal is to demonstrate certain general management principles which can improve patient outcomes.
\end{abstract}

Keywords: Screwdriver, Penetrating brain injury, Right zygomatic bone, Cranitomy

\section{Background}

Intracranial injuries caused by penetrating foreign bodies are associated with high risks of morbidity and mortality due to relevant infection, seizures, vascular injury and cerebrospinal fluid leakage $[1,2]$. Penetrating brain injury (PBI) in civilian population can be caused by almost all sharp and blunt objects, ranging from knives and scissors to chopsticks and screwdrivers [1]. To date, PBI with a screwdriver is a peculiar accident and is relatively rare [3].

Screwdriver-induced PBI is severe and neglected $[4,5]$. First, due to their length and spiral force, the tip of rigid screwdrivers may be enable to penetrate into the calvarium, and once through the bone, the screwdrivers may pivot around the entry point in the skull and then cause curved intracranial injuries, which are far more serious than the damage of skin surface. Second, if the screwdriver is withdrawn, the small entry wound may be missed by clinical examination, and the seriousness of intracranial injuries may also be overlooked [6]. Therefore, it is relatively complex to manage such injuries owing to their rarity and characteristics.

\footnotetext{
* Correspondence: 30468476@qq.com

Department of Neurosurgery, The Third Affiliated Hospital of Soochow University, Changzhou City 213003, China

In this study, we discussed the clinical presentation and successful management of such an unique injury caused by screwdriver. Our goal is to demonstrate certain general management principles which can improve patient outcomes. It is worth mentioning that this case report strictly adhered to care guidelines.

\section{Case presentation \\ History}

A 54-year-old-female patient, without addictions or comorbidities, was admitted in our emergency department due to assault with a screwdriver in her head and loss of consciousness for $1 \mathrm{~h}$. Her colleagues explained that she suffered the injury hit by shedding electric screwdriver when working in a factory, and the screwdriver penetrated the right zygomatic bone (Fig. 1a). The injury was associated with an immediate coma and bleeding of the right ear. The patient had no history of vomiting or seizures. Owing to the disorder of vital signs, the patient underwent tracheal intubation in emergency department.

\section{Examination}

Neurological examination revealed a Glasgow Coma Scale (GCS) of 3/15. A screwdriver was seen partially penetrating the right cheekbone through a lacerated horizontal 




Fig. 1 a. Clinical picture showing a strewdriver penetrating the right cheekbone. b. Computed tomogram (CT) of brain showing the metallic foreign body with metal artefacts. c-d. CT of head-brain window showing a metallic foreign body passed through the right cheekbone to posterior cranial fossa. e-f. CT scan showing left cerebellar hematoma and subarachnoid hemorrhage

wound of $10 \mathrm{~mm} \times 10 \mathrm{~mm}$. The injury was accompanied by left pupillary dilatation $(5 \mathrm{~mm})$ with disappeared responsive to light, right pupillary dilatation $(3 \mathrm{~mm})$ and unresponsive to light, and bleeding of right ear. There was no evidence of any other injury.

A noncontrast axial computed tomographic (CT) scan showed a metallic foreign body passing through the right cheekbone to posterior cranial fossa, and a linear structure extending from right temporal bone, petrous bone, middle cranial fossa, posterior fossa to left occipital bone (Fig. 1b-c). Meanwhile, left cerebellar hematoma and subarachnoid hemorrhage were also demonstrated in CT scan (Fig. 1e-f). Because of the clinical status of the patient, a digital subtraction angiogram could not be performed.

\section{Operation}

The patient was emergently taken to the operating room (OR) and immediately given broad-spectrum antibiotic coverage with penicillin ( $3.75 \mathrm{~g}$, ivgtt). The patient was given a right cheekbone craniectomy and removal of the foreign body at supine position. $6 \mathrm{~cm}$ long straight incision was adopted with the entry point as the center. Craniotomy was performed along the skin incision, during which the screwdriver came along and was removed (Fig. 2a). Afterwards the wound was debrided, cleaned, and closed. Brain CT scan was made again and revealed little hematoma of screwdriver tract, a left cerebellar contusion with about $15 \mathrm{ml}$ hematoma, obvious compression of the fourth ventricle and brain stem, and intraventricular hemorrhage (Fig. 2b-d). Then right frontal puncture was

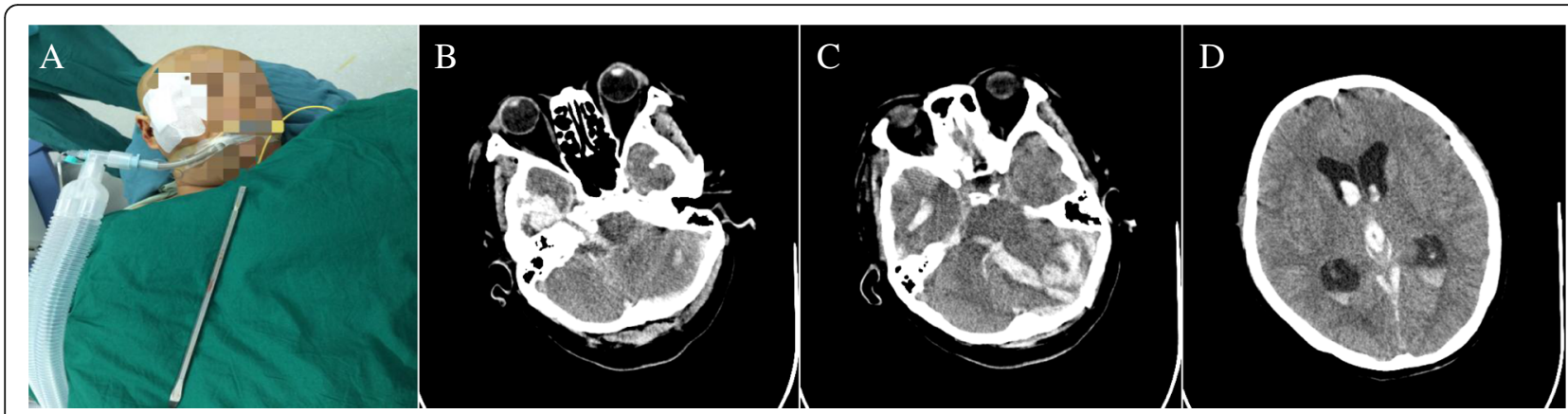

Fig. 2 a. Clinical picture showing the strewdriver removed surgically. b. CT of head-brain window showing hematoma of strewdriver tract. c. CT scan showing a left cerebellar contusion with hematoma and obvious compression of the fourth ventricle and brain stem. $\mathbf{d}$. CT scan showing high density corrosion cast of the ventricular system 
immediately performed, followed by drainage of cerebrospinal fluid (CSF), which was as higher as $20 \mathrm{mmHg}$, to achieve maximum brain relaxation. The patient was then repositioned, placed in prone position and given evacuation of posterior fossa hematoma. The incision was made along the median line, about $16 \mathrm{~cm}$, then skin and muscle flap were separated and the skull was exposed. A $6 \mathrm{~cm} \times$ $5 \mathrm{~cm}$ flap was removed from occipital bone and about $15 \mathrm{ml}$ of subdural hematoma was evacuated. Contusive cerebellar tissues and sutured dura were removed. The patient was then transferred to the neurosurgery intensive care unit (NICU) for postoperative management.

\section{Postoperative outcome}

On post-operative day (POD) 2, brain CT showed smaller posterior fossa hematoma. Meanwhile, external ventricular drainage tube was also placed to replace CSF and prevent obstructive hydrocephalus (Fig. 3a-b). However, the patient suffered persistent coma, with GCS of $4 / 15$, complicated with pulmonary infection in the subsequent 3 days. Tracheotomy was performed to open the airway and control infection. Meanwhile, the patient received aggressive broadspectrum antibiotics which were maintained for 14 days. Intravenous sodium valproate was given for prophylaxis against posttraumatic seizures. No other serious complications were reported after symptomatic supportive measures such as anti-infection treatments and nerve nutrition. The patient's GCS score was improved to 12 on POD 42. Postoperative brain CT scan (Fig. 3c-d) revealed favorable findings. The patient was discharged on POD 60. Postoperative 6-month follow-up evaluation showed normal neurological status of the patient, without further seizures.

\section{Discussion}

PBI involving foreign bodies is less prevalent than closed head injuries but often causes a worse prognosis. To date, there is no standardized management for such injuries because different injury patterns share with each scenario. However, some general management principles can be applied to almost any case to improve patient outcomes.

First is the preoperative examination. Head CT is the most sensitive imaging modality for PBI, to identify the extent of bone and parenchymal injuries and formulate operation plan [7]. In case of suspicion for vascular injury, CT cerebral angiography is also needed to evaluate traumatic aneurysm, which may rapidly develop after PBI [8].

Second is the operative management. In this study, the patient had severe low GCS of 3/15, which could be attributed to 2 points: 1) The characteristics of PBI caused by a screwdriver. 2) The patient presented with posterior fossa hematoma in the early stage, which might oppress brainstem, leading to disorder of vital signs. However, timely and

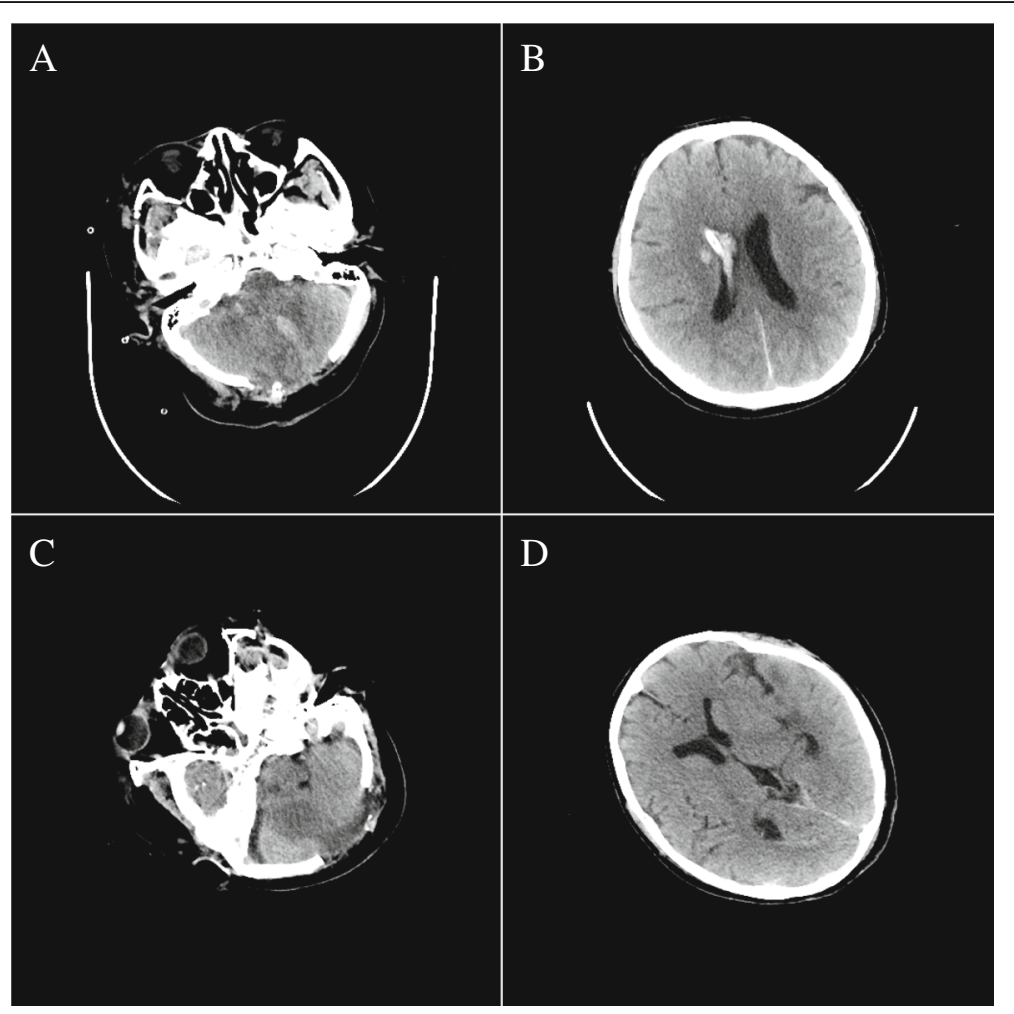

Fig. 3 a-b. CT of brain taken on post-operative day 2, showing smaller posterior fossa hematoma and drainage of CSF. c-d. CT of brain taken on post-operative day 42 , showing complete absorption of haematomas and normal ventricular system 
effective surgical interventions made the patient have a good prognosis [9, 10]. Generally, the goals of surgical intervention for such injury are to: 1) Remove the penetrating object and accompanying necrotic debris around the injury site. According to our study, we recommended removal of the screwdriver through its trajectory with minimum injury. However, in some cases, foreign bodies were removed roughly on the spot, which would lead to bleeding of puncture and poor prognosis $[6,11]$. 2) Eliminate any hematomas developed from the injury. 3) Ensure watertight closure of the dura and prevent CSF leakage [12].

Third is the postoperative management. 1) Prophylactic antibiotics and antiseizure medications are recommended to be applied for the first week $[13,14]$. In this case, we prolonged the use of antibiotics owing to deep penetrating tract and pulmonary infection. 2) For severe PBI patient with postoperative coma and pulmonary infection, tracheotomy could be help to prevent the damage of chronic hypoxia on brain tissue and strengthen the management of respiratory tract. 3) Postoperative imaging and follow-up are important to evaluate complications such as pulmonary infection, delayed intracranial hematoma and posttraumatic hydrocephalus, which can be presented in a delayed mode [8].

However, there are still some limitations in this case report. First, the patient with a metallic foreign body in her head was unable to perform magnetic resonance imaging (MRI). Meanwhile, because of obvious CT imaging artifacts, computed tomography angiography (CTA) imaging and three-dimensional reconstruction could not be used to identify the relationship between metallic foreign body and intracranial vessels or skull. Second, the patient's GCS score on arrival was $3 / 15$ with unstable vital signs and formation of traumatic cerebral hernia. Thus immediate surgical decompression was needed, which kept us from digital subtraction angiography and made us unable to clear intracranial vascular injury. Third, the would tract induced by screwdriver was deep and long, involving multiple lobes,which made it difficult to thorough debridement. Therefore, it may result in residues of foreign bodies or necrotic tissue and increase the risk of infection.

\section{Conclusions}

In conclusion, this is a unique case of penetrating screwdriver injury. Despite the removal of the screwdriver from the intracranial tissue through the orbit, we achieved positive outcomes in this challenging case due to the ability of the neuro trauma unit and the cooperation of multidisciplinary team, involving neurosurgeons, emergency physicians, radiologists and anesthesiologists.

\section{Abbreviations}

CSF: Cerebrospinal fluid; CT: Computer tomography; GCS: Glasgow coma scale; NICU: Neurosurgery intensive care unit; PBI: Penetrating brain injury; POD: Post-operative day

\section{Acknowledgements}

No applicable.

\section{Funding}

No funding was received for the purpose of this case report.

Availability of data and materials

All patient data and clinical approaches adopted are contained in the medical files of The Third Affiliated Hospital of Soochow University. The bibliographic data of reference are available on PubMed and the conclusions are based on the opinion of the expert involved in the this case. The data supporting the conclusions of this article are included within the article and its figures.

\section{Authors' contributions}

$\mathrm{BD}$ was a major contributor in writing and in a review of the manuscript. JS collected patient data and was involved in writing and in a review of the manuscript. YM and JC were involved in patient management and review of the manuscript. All authors read and approved the final manuscript.

\section{Competing interests}

The authors declare that they have no competing interests.

\section{Consent for publication}

Written informed consent was obtained from the patient for publication of this case report and images. A copy of the written consent is available.

Ethics approval and consent to participate

Not applicable.

Received: 26 July 2016 Accepted: 30 November 2016

Published online: 10 January 2017

References

1. Zyck S, Toshkezi G, Krishnamurthy S, Carter DA, Siddiqui A, Hazama A, Jayarao M, Chin L. Treatment of penetrating nonmissile traumatic brain injury. Case series and review of the literature. World Neurosurg. 2016;91: 297-307.6.

2. Mzimbiri JM, Li J, Bajawi MA, Lan S, Chen F, Liu J. Orbitocranial low-velocity penetrating injury: a personal experience, case series, review of the literature, and proposed management plan. World Neurosurg. 2016;87:26-34.

3. de Andrade AF, de Almeida AN, Muoio VM, Marino Jr R. Penetrating screwdriver injury to the brainstem. Case illustration. J Neurosurg. 2006;104(5):853.

4. Meena US, Kataria R, Sharma K, Sardana VR. Penetrating spinal cord injury with screwdriver in situ, leading to Brown-Sequard syndrome. J Neurosci Rural Pract. 2016;7(2):324-7.

5. Bozzeto-Ambrosi P, Costa LF, Azevedo-Filho H. Penetrating screwdriver wound to the head. Arq Neuropsiquiatr. 2008;66(1):93-5.

6. Pavlidis $P$, Karakasi MV, Birbilis TA. Traumatic brain injury due to screwdriver assaults: literature review and case report. Am J Forensic Med Pathol. 2016; 37(4):291-8.

7. Moussa WM, Abbas M. Management and outcome of low velocity penetrating head injury caused by impacted foreign bodies. Acta Neurochir (Wien). 2016;158(5):895-904.

8. Mzimbiri JM, Li J, Xia Y, Yuan J, Liu J, Liu Q. Surviving penetrating brainstem injury by bamboo sticks: rare case reports and a brief review of literature. Neurosurgery. 2016;78(5):E753-60.

9. Das JM, Chandra S, Prabhakar RB. Penetrating brain injury with a bike key: a case report. Ulus Travma Acil Cerrahi Derg. 2015;21(6):524-6.

10. de Holanda LF, Pereira BJ, Holanda RR, Neto JT, de Holanda CV, Giudicissi Filho M, de Oliveira NR, de Oliveira JG. Neurosurgical management of nonmissile penetrating cranial lesions. World Neurosurg. 2016:90:420-9.

11. Ahmad FU, Suri A, Mahapatra AK. Fatal penetrating brainstem injury caused by bicycle brake handle. Pediatr Neurosurg. 2005;41:226-8.

12. Regunath $K$, Awang S, Siti SB, Premananda MR, Tan WM, Haron RH. Penetrating injury to the head: case reviews. Med J Malaysia. 2012;67(6):622-4.

13. Kazim SF, Shamim MS, Tahir MZ, Enam SA, Waheed S. Management of penetrating brain injury. J Emerg Trauma Shock. 2011;4(3):395-402.

14. Xu F, Li J, Sun S, Guo E, Hao S, Hou Z, Leung GK, Liu B. The surgical management of a penetrating orbitocranial injury with a bakelite foreign body reaching the brain stem. Brain Inj. 2013;27(7-8):951-6. 